\title{
ESTUDO DE CORRELAÇÃO ENTRE O CRESCIMENTO DAS MICRO E PEQUENAS EMPRESAS, A EVOLUÇÃO DO PIB BRASILEIRO E O DESEMPREGO
}

\author{
STUDY OF CORRELATION BETWEEN THE GROWTH OF \\ MICRO AND SMALL BUSINESS, THE EVOLUTION OF \\ BRAZILIAN GDP AND UNEMPLOYMENT
}

\section{Recebimento: 19/09/2016- Aceite: 11/11/2016- Publicação: 29/11/2016 Processo de Avaliação: Double Blind Review}

\author{
Erica Vieira ${ }^{1}$ \\ Graduanda em Tecnologia em Gestão Financeira \\ Faculdade de Tecnologia de Osasco Prefeito Hirant Sanazar \\ erica.silva27@fatec.sp.gov.br
}

Fernando de Almeida Santos

Doutor em Ciências Sociais

Professor do Programa de Mestrado de Ciências Contábeis e Atuariais da PUC-SP

Professor integrante do NUPE - Núcleo de Pesquisa ENIAC

fernando@fernandoasantos.com.br

\section{RESUMO}

Este artigo tem por objetivo estudar a correlação entre o crescimento das Micro e Pequenas Empresas, a evolução do PIB nacional e o desemprego. Para o desenvolvimento deste estudo, foi considerado o período de 2003 a 2013, de ambas as variáveis. Assim, foram coletados os dados do PIB e da Taxa Média de Desemprego disponibilizados pelo Instituto Brasileiro de Geografia e Estatística (IBGE), bem como informações das Micro e Pequenas Empresas disponibilizadas pelo SEBRAE. A partir desses dados, foram levantados pontos de análise relevantes para o estudo e aplicado o

\footnotetext{
1 Autor para correspondência: Faculdade de Tecnologia de Osasco Prefeito Hirant Sanazar - Rua Pedro Rissato, 30, Vila dos Remédios, Osasco - SP, Brasil 06296-220.
}

ENIAC Projetos, Guarulhos (SP),V.5, n.2, jun.- dez. 2016 
método estatístico de correlação linear, a fim de verificar a existência ou ausência de uma correlação ou não entre as variáveis de estudo. Os resultados apontam para uma correlação linear entre os objetos de estudo no período avaliado.

PALAVRAS-CHAVE: Crescimento, Desemprego, Micro e Pequenas Empresas, PIB.

\begin{abstract}
This paper aim to study the correlation between the growth of micro and small businesses and the evolution of the national GDP. In order to develop this study, the period from 2003 to 2013 of both variables was considered. Thus, GDP and Unemployment Average Rate data provided by the Brazilian Institute for Geography and Statistics (IBGE) was collected, as well as Micro and Small Businesses information provided by SEBRAE. From that point, points of analysis relevant to the study were collected and the statistical method of linear correlation was applied, in order to verify the existence or absence of a correlation between the study variables. The results indicate a linear correlation between the objects of study in the study period.
\end{abstract}

KEYWORDS: Growth, Unemployment, Micro and Small Businesses, GDP.

\title{
INTRODUÇÃO
}

Segundo dados do SEBRAE (2014), a produção gerada pelas micro e pequenas empresas cresceu de $\mathrm{R} \$ 144$ bilhões para $\mathrm{R} \$ 599$ bilhões de reais, no período de 2001 a 2011, contribuindo para mais de um quarto do PIB brasileiro. Ao mesmo tempo, é possível perceber que a participação das micro e pequenas empresas no PIB nacional atingiu os $27 \%$ em 2011. (SEBRAE 2014).

As micro e pequenas empresas apresentam um atual cenário importante, tanto no que se refere à produção de bens e serviços, quanto para o mercado de trabalho por apresentar estrutura competitiva. (IPEA 2012).

O aumento do PIB indica a que velocidade a economia está crescendo, resultando também como uma melhora potencial do padrão de vida das pessoas. (PARKIN, 2009)

Diante disso, a pergunta de pesquisa é existe correlação entre e o crescimento das micro e pequenas empresas, a evolução do PIB brasileiro e o desemprego?

Assim, após o desenvolvimento deste estudo e verificado que estas duas variáveis apresentam uma correlação, movendo-se de forma acompanhada, torna-se interessante saber que um fator de fomento para o aumento do PIB é o incentivo das

ENIAC Projetos, Guarulhos (SP),V.5, n.2, jun.- dez. 2016 
micro e pequenas empresas. Este quadro dá validade a este estudo por ter considerado a hipótese de relacionar os pequenos e médios negócios como ferramenta direta para o aumento do Produto Interno Bruto.

Esta pesquisa estudou o o crescimento das micro e pequenas empresas, a evolução do PIB brasileiro e o desemprego no período de 2003 a 2013. Para verificar o nível da relação entre as variáveis, utiliza-se o Coeficiente de Correlação Linear visto que este coeficiente de correlação é uma medida para determinar a intensidade da força e direção da relação linear entre duas variáveis. É determinada pelo símbolo $r$. (LARSON e FARBER, 2012).

A seguir a fórmula do coeficiente de correlação de Pearson.

Figura 1 - Fórmula do Coeficiente de Correlação

$$
r=\frac{n \Sigma x y-\Sigma x \Sigma y}{\sqrt{n \Sigma x^{2}-(\Sigma x)^{2}} \sqrt{n \Sigma y^{2}-(\Sigma y)^{2}}}
$$

Fonte dos dados: LARSON E FARBER, 2012 (CRESPO, 2009)

Assim, se o valor de $r$ está próximo a +1 , existe uma correlação linear positiva entre as variáveis x e y. Se o valor de $r$ está próximo a -1, existe uma correlação linear negativa entre as variáveis $\mathrm{x}$ e y. E se o valor de $r$ está próximo a 0 , significa que não há relação linear entre as duas variáveis.

Segundo Schaum (1993), após submeter os dados ao teste de correlação é que se pode medir quão forte é a relação das duas variáveis envolvidas.

Foram coletados os dados do PIB e do desemprego, tendo como fonte o IBGE, e os dados das micro e pequenas empresas foram obtidos em publicações do SEBRAE. Assim, foram estabelecidos os pontos de análise, submetendo os dados das variáveis aos métodos estatísticos, resultando em informações a serem analisadas com o objetivo de responder à pergunta de pesquisa deste trabalho.

\section{MICRO E PEQUENAS EMPRESAS NO BRASIL}

A Lei Complementar $\mathrm{n}^{\circ} 123$, art. $5^{\circ}$, de 14 de dezembro de 2006, institui o Estatuto Nacional da Microempresa e da Empresa de Pequeno Porte. O art. $3^{\circ}$ da Lei 
Complementar define microempresas ou empresas de pequeno porte uma sociedade empresária, simples ou empresa individual com responsabilidade limitada.

A lei define ainda microempresa como a empresa que aufere, dentro do anocalendário, receita bruta inferior ou igual a $\mathrm{R} \$ 360.000,00$ (trezentos e sessenta mil reais). São consideradas empresas de pequeno porte as que possuem receita bruta superior a $\mathrm{R} \$ 360.000,00$ (trezentos e sessenta mil reais) - e igual ou inferior a $\mathrm{R} \$ 3.600 .000,00$ (três milhões e seiscentos mil reais).

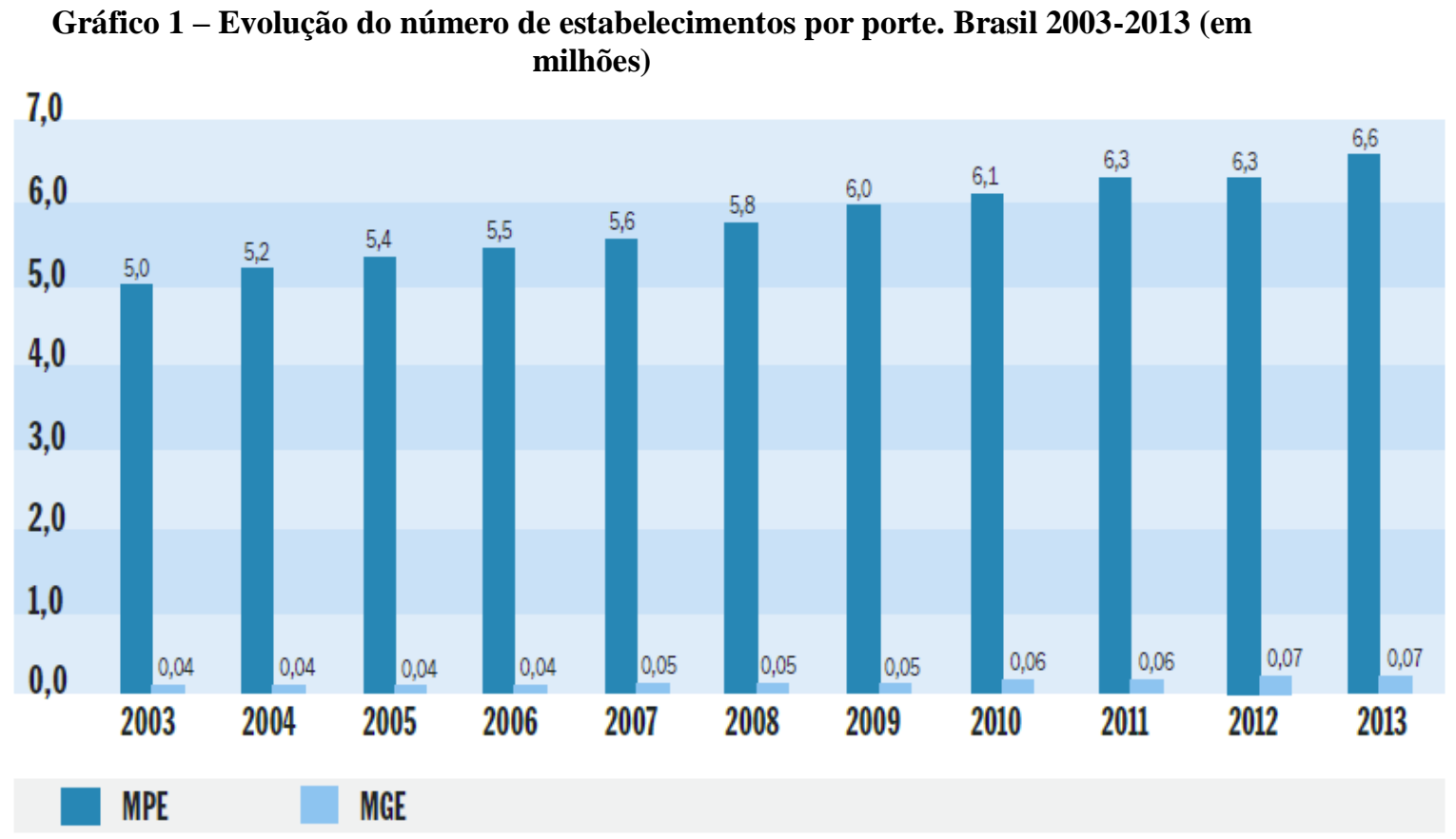

Fonte dos dados: MTE. RAIS. Elaborado pela DIEESE.*Setores considerados: indústria, comércio e serviços

O gráfico 1 demonstra o crescimento das MPEs em relação as Médias e Grandes Empresas - MGE. No início do período, 2003, o Brasil possuía 5 milhões de estabelecimentos, atingindo 6,6 milhões de empresas em 2013, totalizando o aumento de 1,7 milhões de novas MPEs. É possível perceber a disparidade entre o crescimento das MPE's e as MGE durante o período estudado, as MPE's apresentam um crescimento muito superior ao das MGE.

ENIAC Projetos, Guarulhos (SP),V.5, n.2, jun.- dez. 2016 


\section{PRODUTO INTERNO BRUTO - PIB}

"Produto Interno Bruto - PIB é um valor de mercado dos bens e serviços, produzidos em território nacional em determinado período”. (PARKIN, 2009).

"O PIB pode variar dentro de um período de tempo. Esta variação tem por motivo a mudança de quantidade disponível de recursos da economia". (DORNBUSCH, FISCHER e STARTZ, 2009). Assim, "a taxa de crescimento da economia é a taxa à qual o produto interno bruto cresce." (DORNBUSCH, FISCHER e STARTZ, 2009).

Tabela 1- Fechamento do PIB. 2003 - 2013. (em bilhões de reais).

\begin{tabular}{cc}
\hline Ano & Valores Correntes \\
\hline 2003 & 1.720 .069 \\
2004 & 1.958 .705 \\
2005 & 2.171 .736 \\
2006 & 2.409 .803 \\
2007 & 2.718 .032 \\
2008 & 3.107 .531 \\
2009 & 3.328 .174 \\
2010 & 3.886 .835 \\
2011 & 4.374 .765 \\
2012 & 4.713 .096 \\
2013 & 5.157 .569 \\
\hline
\end{tabular}

Fonte de dados: IBGE Séries Estatísticas. Elaborada pelos Autores, 2016.

Na tabela 1, é apresentado o fechamento anual do PIB brasileiro em bilhões de reais no período estudado.

\section{DESEMPREGO}

A seguir, apresenta-se o Índice de desemprego.

"Desemprego é o conjunto de pessoas acima de uma determinada idade que estão sem trabalho, disponíveis para trabalhar." (INTERNATIONAL LABOUR OFFICE - ILO).

"O que mais caracteriza o desemprego é a desigualdade na distribuição de renda na sociedade, além de causar queda do padrão de vida do desempregado”. (SACHS e LARRAIN, 2000). 
Tabela 2- Taxa média de desemprego. 2003 - 2013. (\%)

\begin{tabular}{cr}
\hline Ano & \% \\
\hline 2003 & 12,4 \\
2004 & 11,5 \\
2005 & 9,9 \\
2006 & 10 \\
2007 & 9,3 \\
2008 & 7,9 \\
2009 & 8,1 \\
2010 & 6,7 \\
2011 & 6 \\
2012 & 5,5 \\
2013 & 5,4 \\
\hline Fonte de dados: IBGE 2014. \\
Elaborada pelos Autores
\end{tabular}

A partir dos dados da tabela 2, é possível verificar que, no período analisado, a taxa média apenas declinou, com exceção dos anos de 2006 e 2009 quando a taxa teve um aumento, porém, não grande. Portanto, foi um resultado positivo econômico para o período já que a queda da taxa média se deu em mais de 50\% entre 2003 a 2013.

\section{ANÁLISE E DISCUSSÃo}

Neste tópico apresentam-se os resultados após as aplicações estatísticas dos dados coletados no período de 2003 a 2013, bem como, também, as análises e interpretações a partir dos resultados obtidos apresentados por meio de gráficos. Demonstra-se, portanto a relação entre o crescimento das MPE's e o aumento do PIB nacional.

\subsection{Relação entre as micro e pequenas empresas e o PIB.}

A finalidade deste estudo consiste em examinar a relação entre o aumento da quantidade de micro e pequenas empresas anualmente e a evolução do PIB nacional. Para realizar o estudo deste grau de relação, utiliza-se o instrumento estatístico correlação. A seguir, apresentam-se os dados em um diagrama de dispersão. É um diagrama que auxilia de forma subjetiva, porém útil, avistar a correlação existente. (CRESPO, 2009).

O diagrama de dispersão é representado em um sistema cartesiano ortogonal por meio de pares ordenados $\left(\mathrm{x}_{\mathrm{i}}, \mathrm{y}_{\mathrm{i}}\right)$ onde a variável independente (exploratória) $\mathrm{x}$ é apresentada na horizontal, e a variável dependente (resposta) y é apresentada na vertical. (LARSON e FARBER, 2012). 
Assim, estabeleceram-se os dados das MPE's como variável x e o PIB como variável y. O diagrama de dispersão pode definir a existência de uma correlação linear entre as variáveis e também seus diversos tipos. (LARSON e FARBER, 2012).

Gráfico 2- Relação entre as MPE's e o PIB . 2003 - 2013

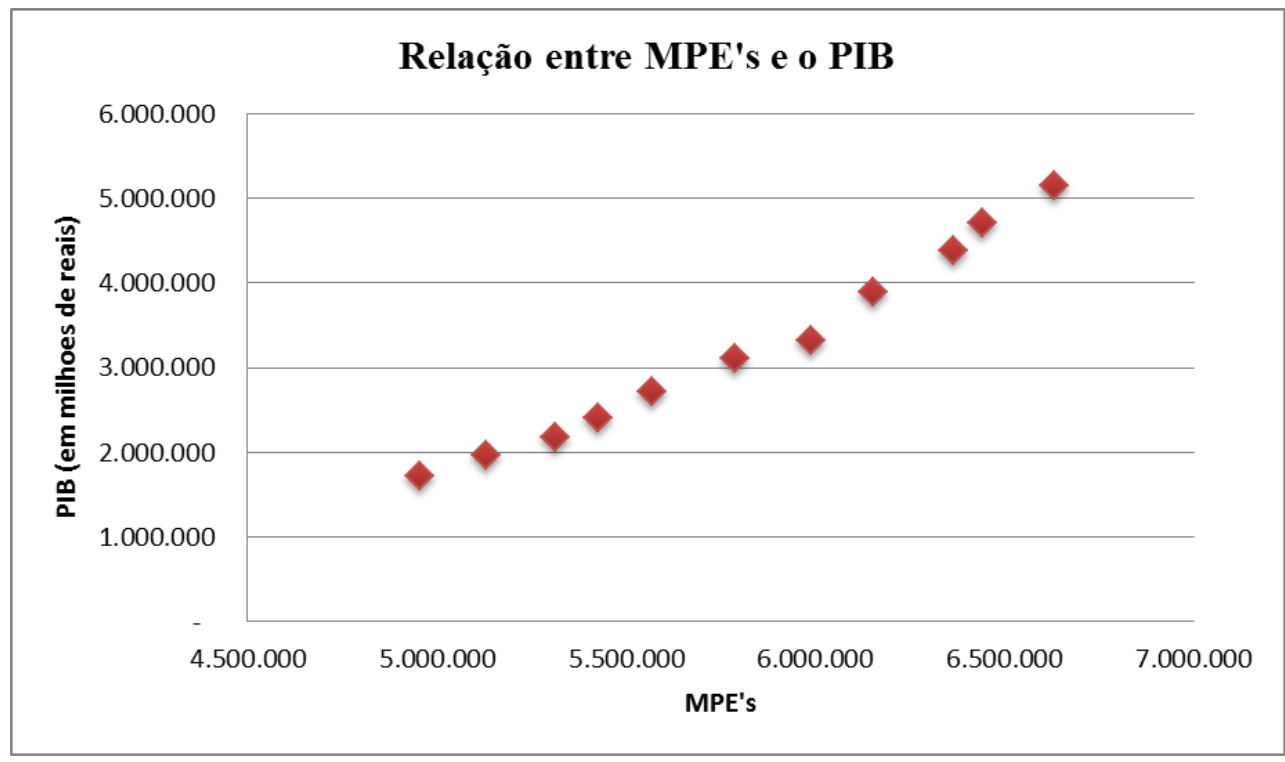

Fonte dos Dados: IBGE (2015) e SEBRAE (2014). Elaborado pelos Autores.

$$
r=0,9911
$$

Após essa apresentação, conforme o gráfico 2, é possível verificar que aparentemente há correlação linear entre o aumento das Micro e Pequenas empresas e o PIB nacional no período analisado, visto que apresentados no diagrama de dispersão as variáveis refletiram um crescimento acompanhado.

O coeficiente de correlação é de 0,9911 entre o crescimento das micro e pequenas empresas e o PIB. Isso significa que existe uma forte correlação entre as duas variáveis, quase perfeita. Portanto, pode-se afirmar que o aumento das MPE's no Brasil possibilitou o aumento do PIB.

\subsection{Relação entre a Participação das Regiões Brasileiras no PIB e o PIB.}

Com o objetivo de alcançar um estudo mais minucioso é que se realizou uma análise entre a participação das cinco regiões brasileiras no PIB separadamente e o PIB nacional. A região que apresenta a maior quantidade de MPE's é a região sudeste: apenas no estado de São Paulo ultrapassou os 2 milhões de MPE's.

ENIAC Projetos, Guarulhos (SP),V.5, n.2, jun.- dez. 2016 
Gráficos 3 - Relação entre a Quantidade de MPE's na região Nordeste e o PIB em volume. 2003 - 2013

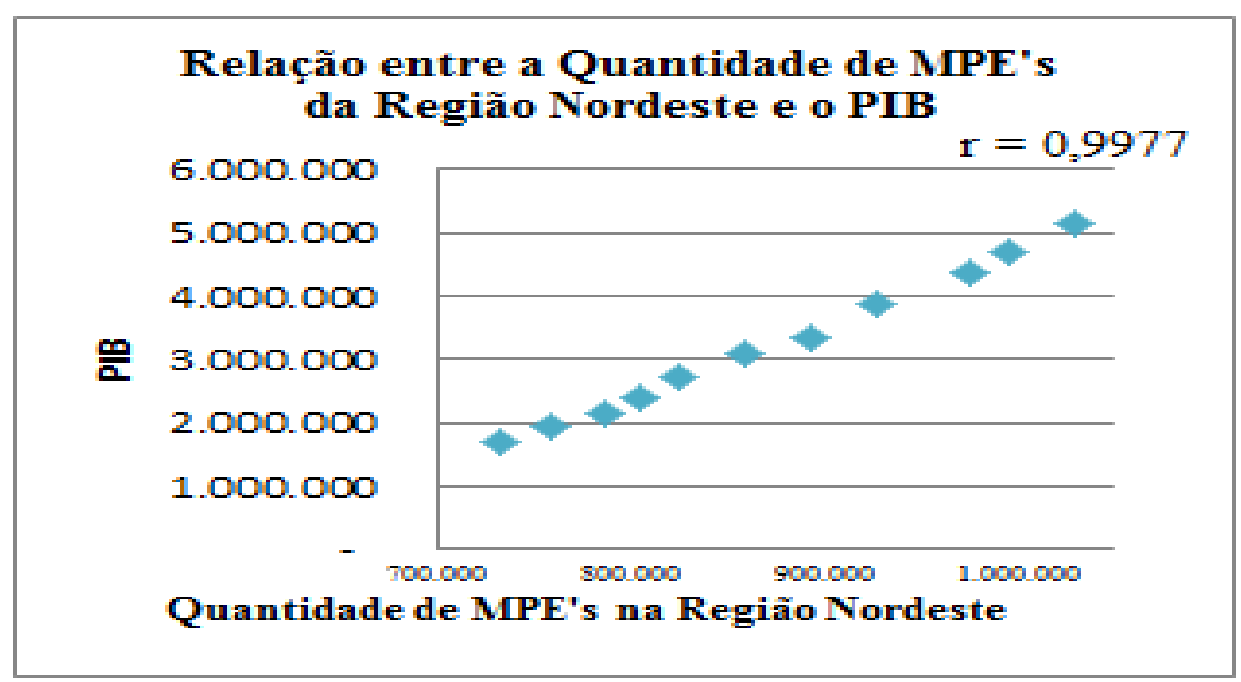

Fonte dos Dados: IBGE (2015) e SEBRAE (2014). Elaborado pelos Autores.

O gráfico de dispersão aponta para uma relação linear entre as variáveis. Após o cálculo do coeficiente de Person encontrado da região nordeste foi de $r=0,9977$. Portanto, existe uma forte correlação entre a quantidade de Micro e pequenas empresas desta região e o PIB.

\section{Gráficos 4 - Relação entre a Quantidade de MPE's na região Sudeste e o PIB em volume. 2003 - 2013}

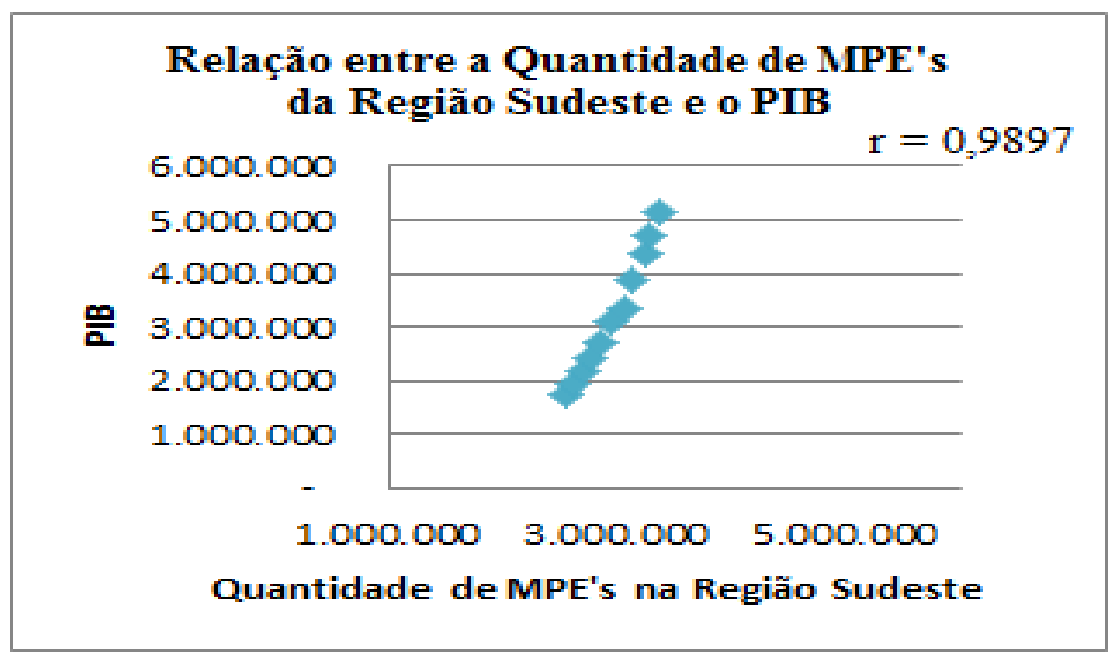

Fonte dos Dados: IBGE (2015) e SEBRAE (2014). Elaborado pelos Autores.

O gráfico de dispersão com quantidade de MPE's da região Sudeste aponta também para uma correlação: após o cálculo do valor de $r$ que é 0,9897 é confirmada a forte correlação.

ENIAC Projetos, Guarulhos (SP),V.5, n.2, jun.- dez. 2016 


\section{Gráficos 5 - Relação entre a Quantidade de MPE's na região Centro Oeste e o PIB}

em volume. 2003 - 2013

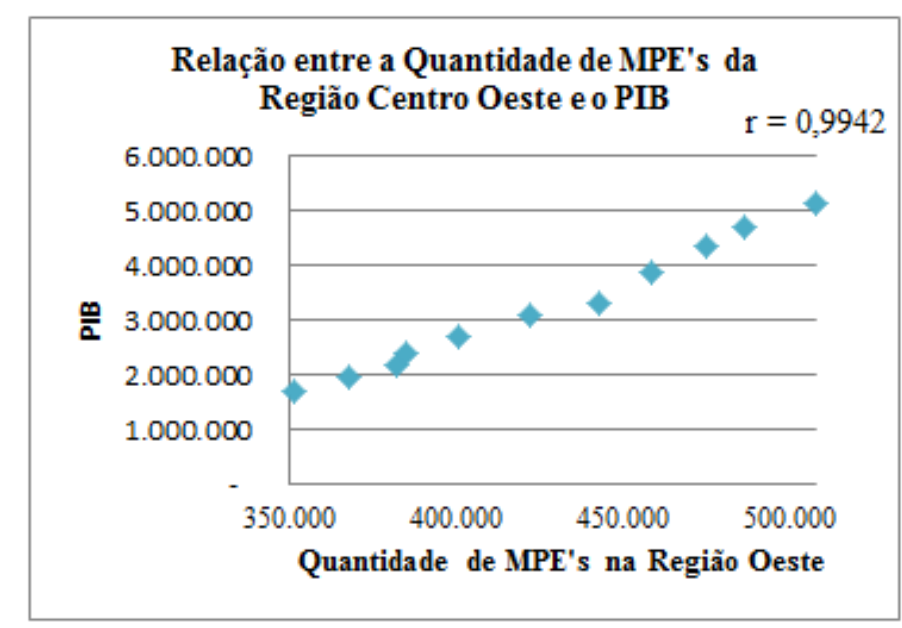

Fonte dos Dados: IBGE (2015) e SEBRAE (2014). Elaborado pelos Autores.

O gráfico representando a relação entre a quantidade de MPE's na região Centro Oeste e o PIB revela a existência de uma correlação. Esta correlação pode ser confirmada através do resultado do coeficiente de correlação que apresentou grande proximidade a 1 .

\section{Gráficos 6 - Relação entre a Quantidade de MPE's na região Sul e o PIB em} volume. 2003 - 2013

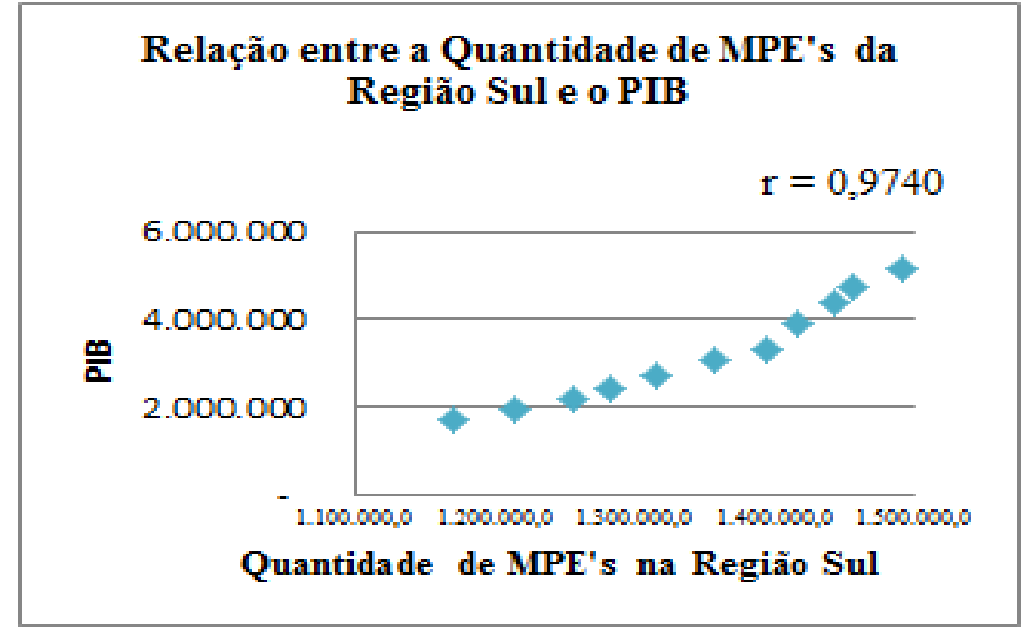

Fonte dos Dados: IBGE (2015) e SEBRAE (2014). Elaborado pelos Autores.

O gráfico 6 apresenta um gráfico de dispersão com uma inclinação crescente. Essa inclinação é confirmada através do valor de $r$ que é próximo a 1.

ENIAC Projetos, Guarulhos (SP),V.5, n.2, jun.- dez. 2016 


\section{Gráficos 7 - Relação entre a Quantidade de MPE's na região Norte e o PIB em volume. 2003 - 2013}

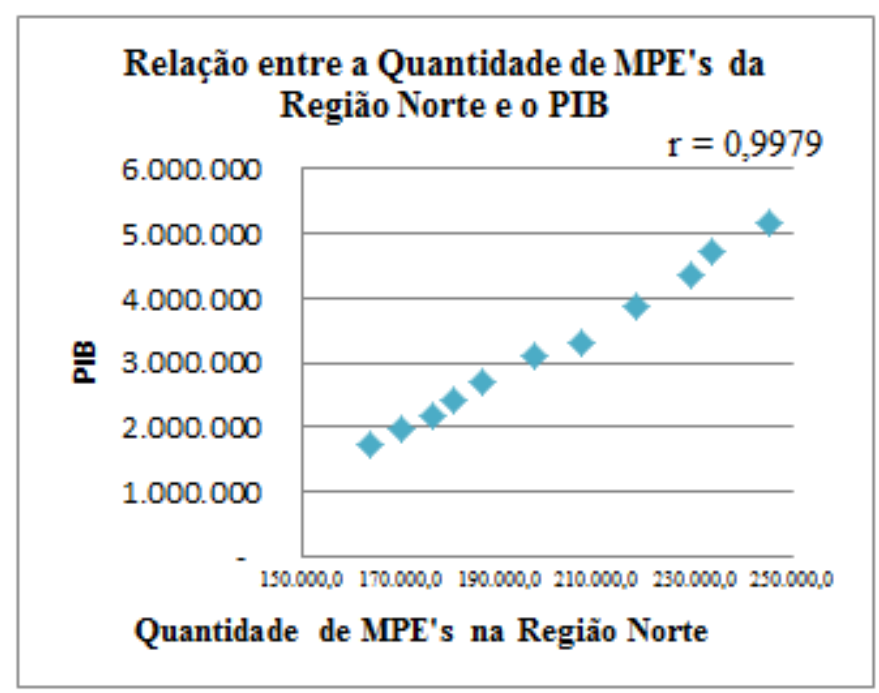

Fonte dos Dados: IBGE (2015) e SEBRAE (2014). Elaborado pelos Autores.

Os gráficos de 3 a 7 apresentam dispersão com a quantidade de MPE's por região. É possível observar rapidamente que há uma forte linearidade entre o crescimento das micro e pequenas empresas e o PIB já que cada região apresentou uma tendência crescente no gráfico e seu coeficiente de correlação das cinco regiões manteve-se sempre próximo a 1.

Vale destacar que, dentre as cinco regiões, o coeficiente de correlação mais próximo de 1 foi nas regiões Norte, Nordeste e Centro-Oeste. Curiosamente, são regiões que apresentam uma quantidade menor de grandes empresas. A partir daí, é possível perceber a importância dos pequenos negócios nessas regiões brasileiras.

\subsection{Relação entre a Participação das MPE's por atividade econômica e o PIB .}

O Brasil desenvolve algumas atividades econômicas. Este tópico dispõe uma relação entre a participação dos setores econômicos no PIB e as MPE's. Com isso, abordaremos apenas a participação do PIB nos setores de serviços, comércio e indústria apresentando, a princípio, também sua relação com as MPE's. 
Gráfico 8- Relação entre o \% de participação da Indústria no PIB e o PIB em volume. $2003-2013$

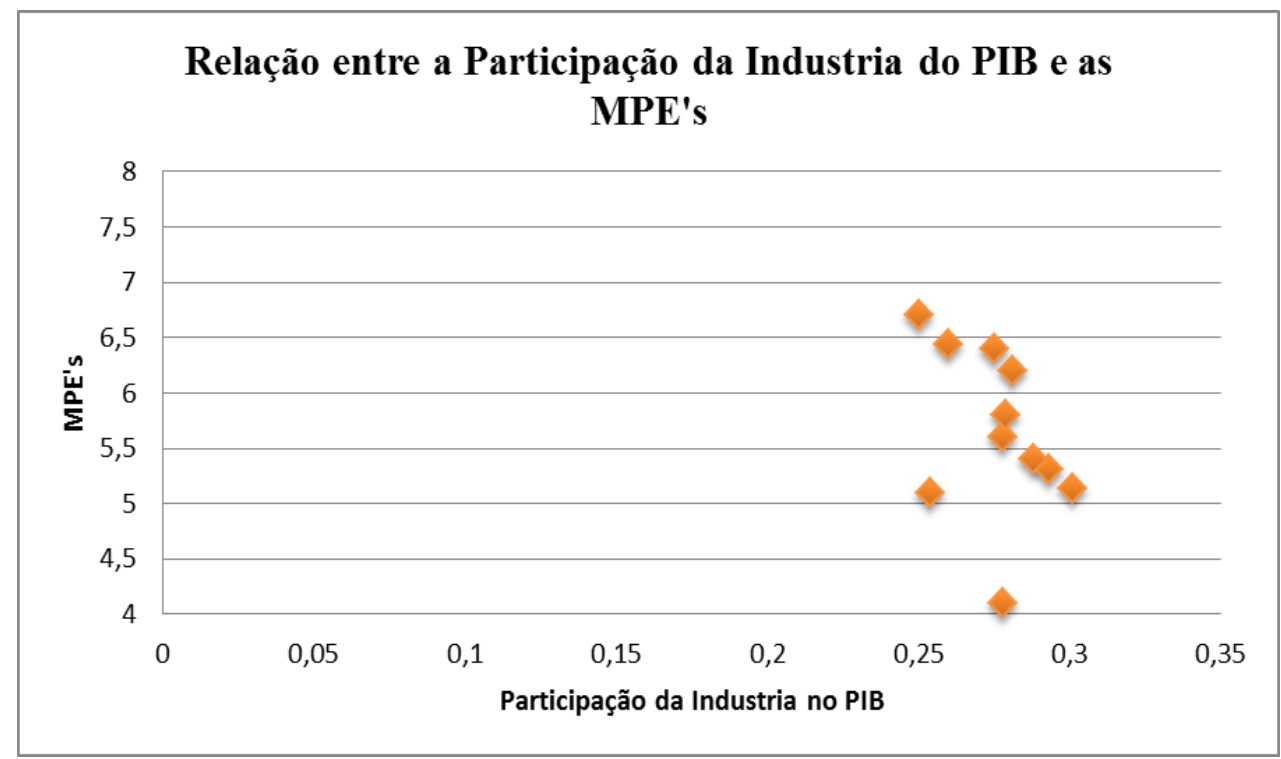

Fonte dos Dados: IBGE (2015) e SEBRAE (2014). Elaborado pelos Autores.

$$
r=-0,4179
$$

O gráfico 8 demonstra que as variáveis "Participação da indústria no PIB" e as "Micro e pequenas" empresas, no período analisado, aparentemente, não apresentam uma relação linear.

Gráfico 9 - Relação entre o \% de participação do Comércio no PIB e o PIB em volume. 2003 - 2013

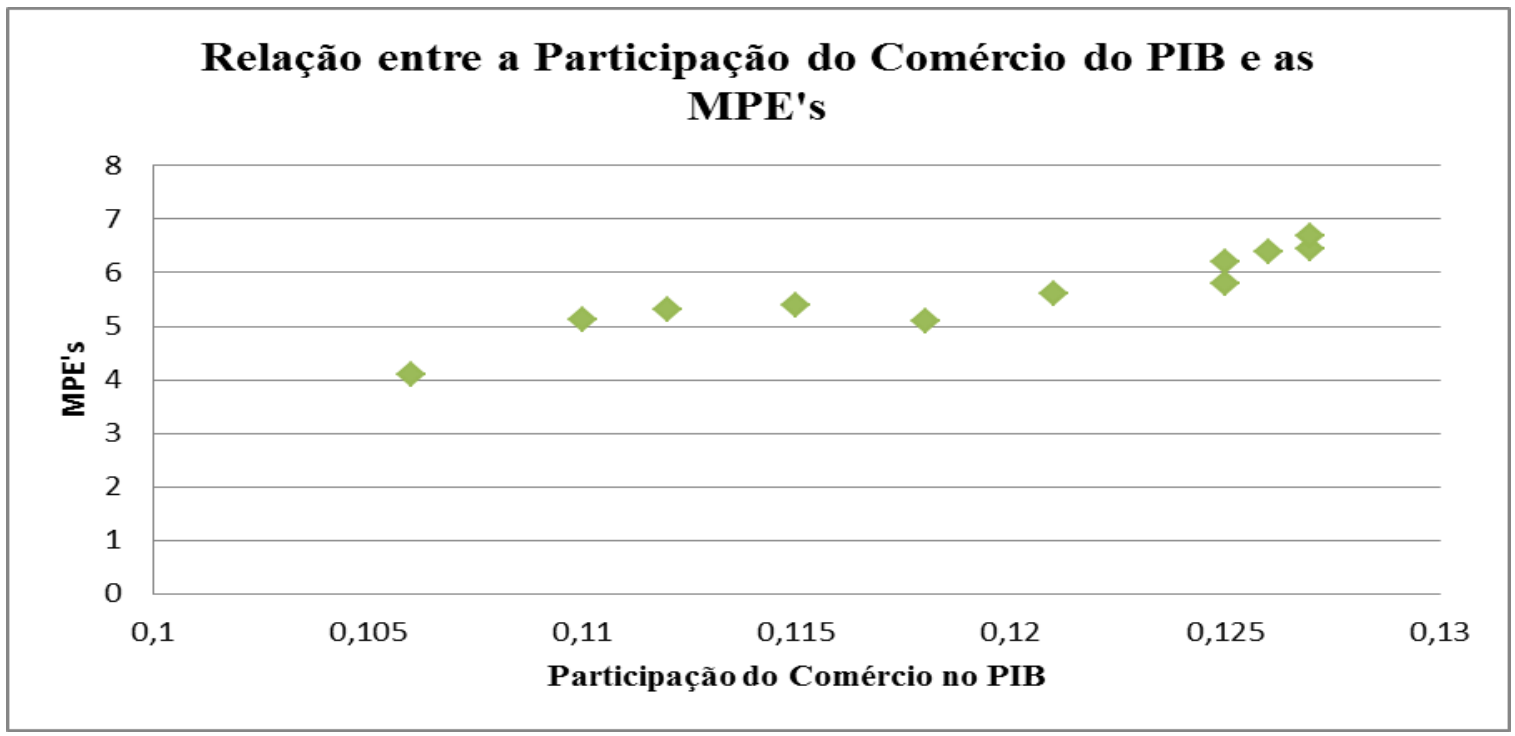

Fonte dos Dados: IBGE (2015) e SEBRAE (2014). Elaborado pelos Autores.

$$
r=0,9225
$$

Outro setor do mercado brasileiro é o segmento do comércio. No gráfico 9, o diagrama de dispersão apresenta uma relação linear entre as variáveis de estudo já que

ENIAC Projetos, Guarulhos (SP),V.5, n.2, jun.- dez. 2016 
este apresenta uma tendência crescente. Portanto, aponta que a evolução do comércio no PIB cresceu proporcionalmente as MPE's.

O coeficiente de correlação é forte e positivo, de 0,9225 , confirmando a força da relação entre a participação do comércio no PIB e as micro e pequenas empresas.

Gráfico 10 - Relação entre o \% de participação do Comércio no PIB e o PIB em volume. 2003 - 2013

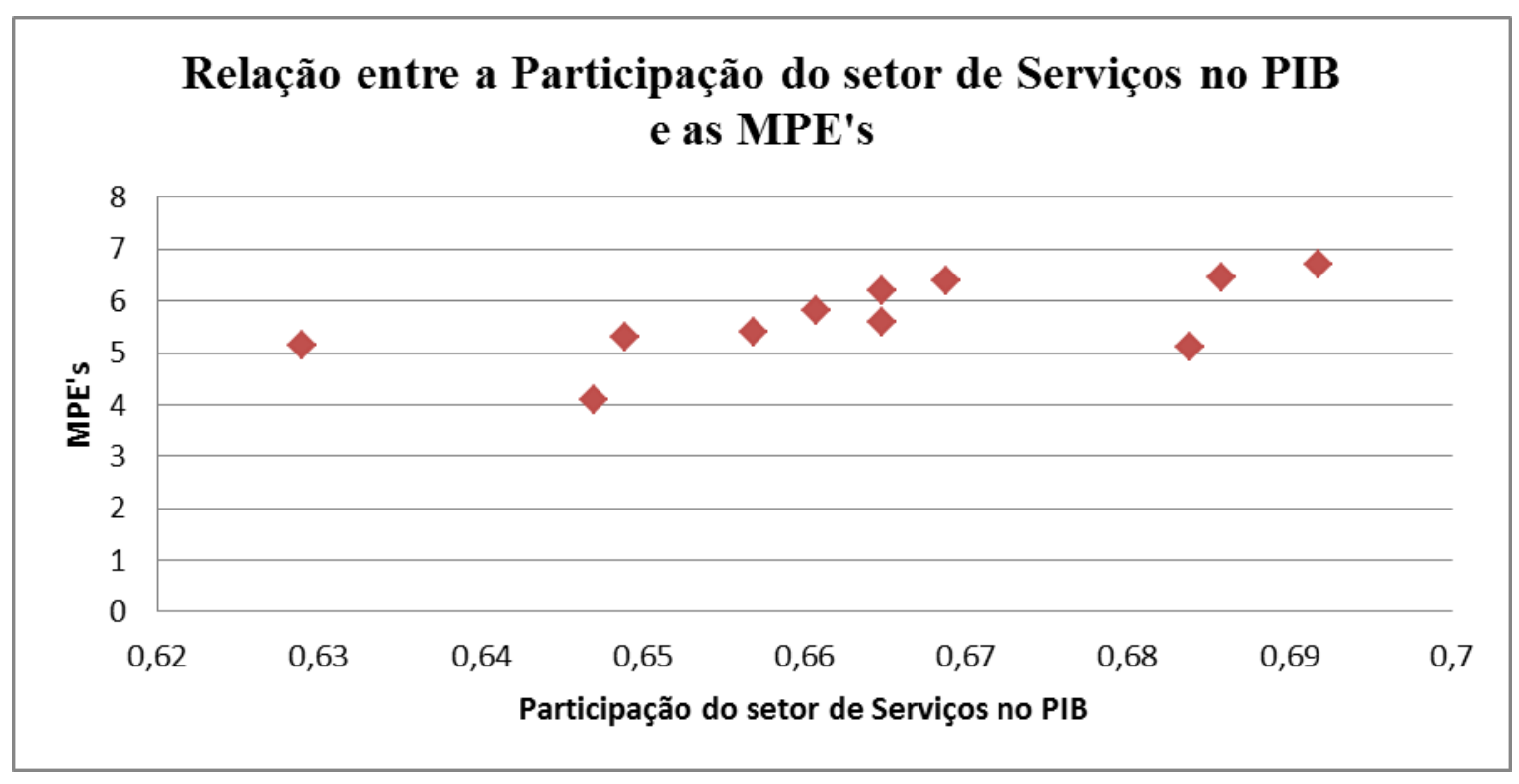

Fonte dos Dados: IBGE (2015) e SEBRAE (2014). Elaborado pelos Autores.

$$
r=0,6391
$$

A partir do coeficiente de correlação percebe-se que existe sim uma relação negativa e fraca entre as variáveis.

A partir do gráfico de dispersão 10, é possível perceber uma correlação linear moderada entre a participação do setor de serviços no PIB e as MPE's. O gráfico apresenta uma tendência não muito crescente ou delineada. O coeficiente é 0,6391, portanto, a correlação não é tão forte, porém, também não é fraca. Trata-se de uma correlação moderada.

\subsection{Taxa de Desemprego e PIB}

A taxa de desemprego apresenta-se como um importante indicador, portanto, com o objetivo de verificar uma possível correlação entre as variáveis taxa média de desemprego e o PIB é que é apresentada no gráfico 13 a relação entre estes medidores.

ENIAC Projetos, Guarulhos (SP),V.5, n.2, jun.- dez. 2016 
Gráfico 11 - Relação entre a taxa média de Desemprego e o PIB. 2003 a 2013.

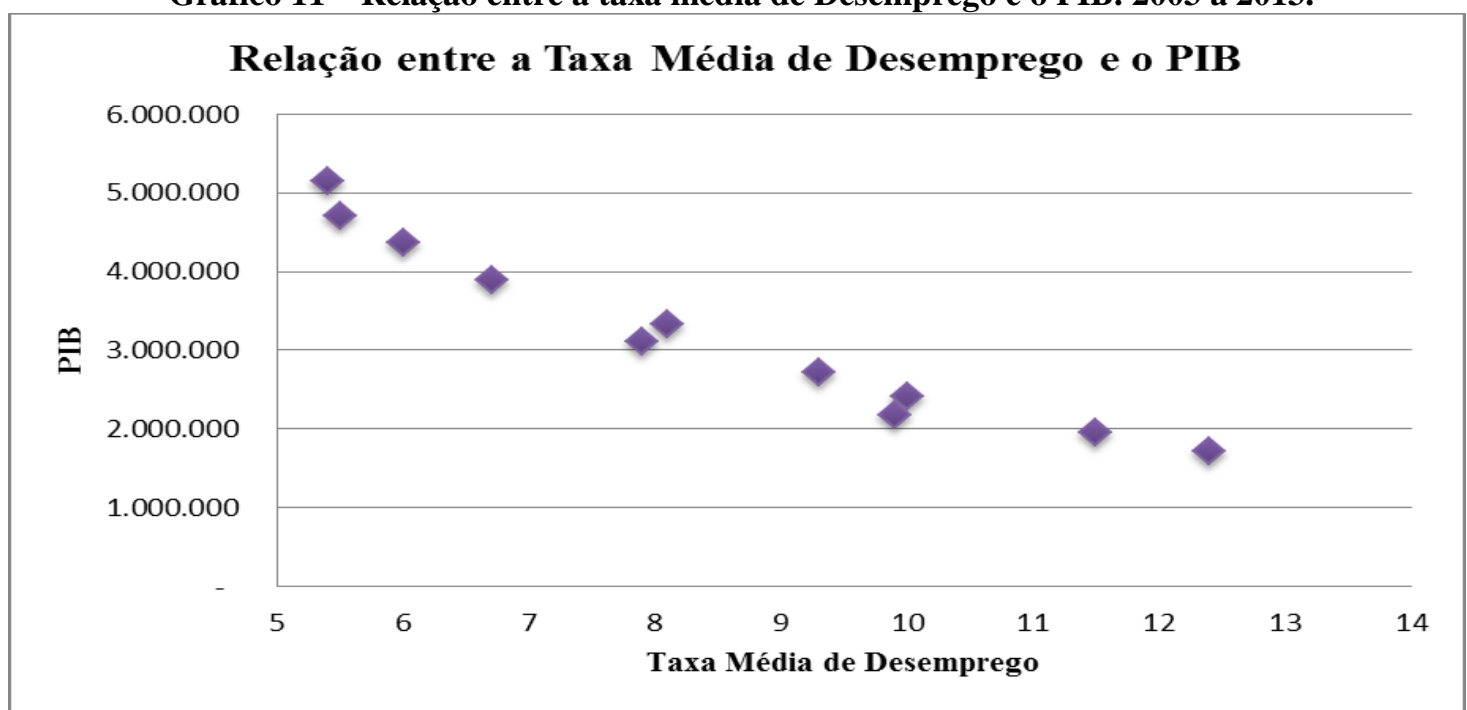

Fonte dos Dados: IBGE (2015) e SEBRAE (2014). Elaborado pelos Autores.

$$
r=-0,9725
$$

O gráfico 11 possibilita visualmente perceber que existe uma forte correlação linear negativa entre as variáveis. É possível perceber uma tendência decrescente. Nesta relação, enquanto a taxa de desemprego declinava, o PIB evoluía, indicando uma relação inversamente proporcional entre as variáveis. O valor de $\mathrm{r}$ é $-0,9725$ indicando uma forte correlação negativa entre a taxa de desemprego e o PIB nacional.

\subsection{Taxa Média de Desemprego e as MPE's}

É importante para este estudo comparar variáveis que, ao longo do tempo, vão se comportando de forma inversamente proporcional como a taxa média de desemprego e as MPE's, principalmente quando um (ou uma variável) mede a falta de empregos e o outro (a outra) representa a quantidade do aumento de pequenas empresas.

ENIAC Projetos, Guarulhos (SP),V.5, n.2, jun.- dez. 2016 
Gráfico 12 - Relação entre a taxa média de Desemprego e as MPE's. 2003 a 2013.

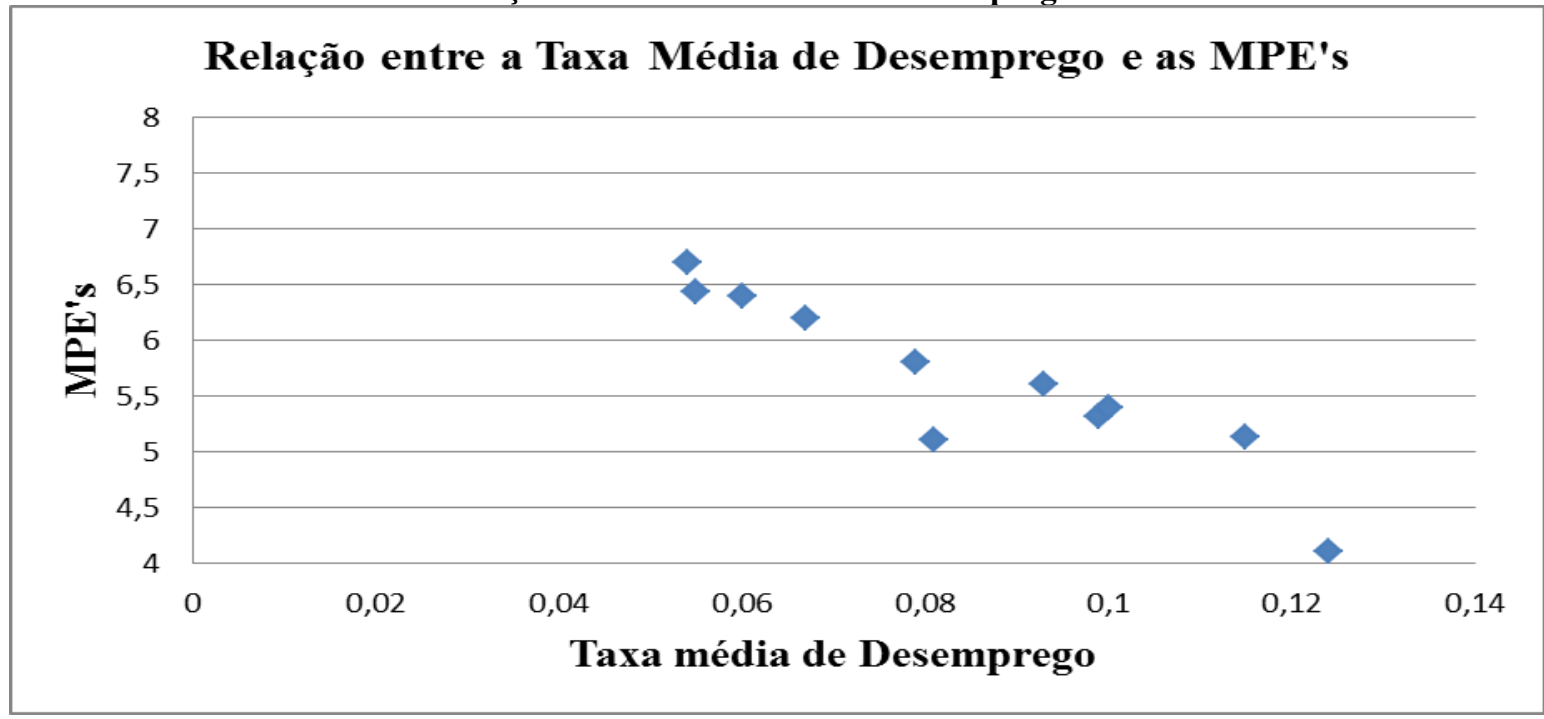

Fonte dos Dados: IBGE (2015) e SEBRAE (2014). Elaborado pelos Autores.

$$
r=-0,9243
$$

O gráfico 12 apresenta a relação entre a taxa média de desemprego e as MPE's. Visualmente, é possível perceber uma tendência decrescente, indicando uma correlação negativa. A posteriori, após o cálculo do coeficiente de relação de -0,9243, é confirmada a existência de uma forte correlação negativa. Assim, à medida que a taxa de desemprego declinava, o PIB aumentava, indicando uma relação inversamente proporcional.

\section{CONSIDERAÇÕES}

Por conseguinte, após a apresentação a priori dos dados das variáveis mostrados no diagrama de dispersão, é calculado o coeficiente de correlação.

Para realizar a medida da correlação linear com mais precisão que a do diagrama de dispersão, é preciso calcular o coeficiente de correlação (LARSON e FARBER, 2012),

O coeficiente de correlação é uma medida para determinar a intensidade da força e da direção da relação linear das duas variáveis. É determinada pelo símbolo $r$ (LARSON e FARBER, 2012).

Para o desenvolvimento do estudo, foram utilizados 22 dados, 11 dados de cada variável. Sobre o PIB, foi usado valor corrente em milhões de reais. Conforme os dados do estudo, foi elaborado o quadro 1. 


\section{Quadro 1 - Resultados}

\begin{tabular}{|c|c|}
\hline \multirow{3}{*}{$\begin{array}{l}\text { Relação quantidade de } \\
\text { MPE's e o PIB }\end{array}$} & \\
\hline & $R$ \\
\hline & $911^{0,9}$ \\
\hline \multirow{2}{*}{$\begin{array}{c}\text { Taxa Média de Desemprego } \\
\text { e o PIB }\end{array}$} & $R$ \\
\hline & 0,9725396 \\
\hline \multirow{2}{*}{$\begin{array}{l}\text { Taxa Média de Desemprego } \\
\text { e as MPE's }\end{array}$} & $R$ \\
\hline & 0,924307 \\
\hline
\end{tabular}

\begin{tabular}{|c|c|c|c|c|c|}
\hline \multirow{3}{*}{$\begin{array}{l}\text { Quantidade de MPE's nas } \\
\text { Regiões Brasileiras e o PIB }\end{array}$} & $\begin{array}{l}\text { SU } \\
\text { DESTE }\end{array}$ & $\begin{array}{l}\text { NOR } \\
\text { DESTE }\end{array}$ & $\mathbf{U L}^{\mathbf{S}}$ & ORTE & ESTE $^{\mathrm{O}}$ \\
\hline & $R$ & $r$ & $R$ & $r$ & $R$ \\
\hline & $\begin{array}{r}0,9 \\
897236 \\
\end{array}$ & $\begin{array}{r}0,997 \\
767933 \\
\end{array}$ & $\begin{array}{r}0, \\
9740084 \\
\end{array}$ & $\begin{array}{r}0 \\
, 9979 \\
\end{array}$ & $\begin{array}{r}0 \\
, 994289 \\
\end{array}$ \\
\hline
\end{tabular}

\begin{tabular}{|c|c|c|c|}
\hline \multirow{3}{*}{$\begin{array}{l}\text { Participação dos setores de } \\
\text { atividade econômica no PIB e as } \\
\text { MPE's }\end{array}$} & DÚSTRIA $^{\text {IN }}$ & ÉRCIO COM & RVIÇOS \\
\hline & $R$ & $r$ & $R$ \\
\hline & - & $\begin{array}{r}0,922 \\
531256\end{array}$ & $\begin{array}{r}0 \\
6391867\end{array}$ \\
\hline
\end{tabular}

Considerando que o coeficiente de correlação ou o valor de $\mathrm{r}$ tem como amplitude +1 a -1 , temos que a relação entre a quantidade de MPE's e o PIB é uma correlação linear positiva, quase perfeita, já que o valor de r é 0,9911.

A taxa média de desemprego e o PIB apresentam também uma forte correlação linear negativa com seu valor de $\mathrm{r}-0,9725$, bem como a taxa média de Desemprego e as MPE's com r -0,924307.

A quantidade de micro e pequenas empresas por regiões brasileiras e o PIB apresentaram também uma forte correlação linear positiva

Entre a relação da participação dos setores de atividade econômica no PIB e as micro e pequenas empresas, apenas o comércio apresentou uma forte correlação linear com seu valor de r 0,9225. Entre os demais setores estudados, o setor de serviços apresentou o valor de $\mathrm{r}$ 0,6391, apresentando mesmo assim uma correlação. $\mathrm{O}$ setor de indústria apresentou também uma considerável correlação linear negativa. 


\section{CONSIDERAÇÕES FINAIS}

O desempenho positivo das micro e pequenas empresas, nos últimos anos, foi acompanhado pelo PIB que, no mesmo período, apresentou resultado significativo.

Consequentemente, neste período de desenvolvimento, o desemprego apresentou sempre queda. Todos esses fatos unidos promoveram um cenário favorável ao crescimento econômico nacional.

Portanto, diante dos resultados obtidos, respondendo à pergunta de pesquisa e a partir dos pontos de análise, pode-se concluir que existe relação entre o Produto Interno Bruto e as micro e pequenas empresas no período estudado com o coeficiente de correlação $r$ de 0,9911, uma correlação quase perfeita. Logo, o PIB apresentou um crescimento acompanhado pelo crescimento das micro e pequenas empresas no período analisado.

Em contrapartida, e favoravelmente, à medida em que a taxa de desemprego declinava, o PIB mantinha seu crescimento, apresentando um valor de $\mathrm{r}$ de $-0,9725 \mathrm{o}$ que indica uma forte correlação negativa. Com isso, conclui-se que ambos se desenvolveram de maneira inversamente proporcional, assim como a taxa de desemprego e o crescimento das micro e pequenas empresas.

O crescimento das MPE's por região, também, apresentou uma forte correlação positiva com o PIB, mantendo seu coeficiente de correlação sempre próximo de 1 .

A participação dos setores de atividade econômica no PIB, como a indústria, apresentou uma correlação negativa moderada, apontando que, entre o crescimento das micro e pequenas empresas e o setor de serviços, não há uma relação maior. $O$ comércio, por sua vez, apresentou uma forte correlação com o crescimento das MPE's, e a participação da indústria no PIB e as MPE's não apresentou uma correlação linear. No geral, entre as variáveis estudadas, a maioria apresentou valores de $\mathrm{r}$ expressivos, endossando a realidade de que o crescimento das micro e pequenas empresas teve grande importância no aumento do PIB nacional, podendo-se até utilizar o desenvolvimento de MPE's como ferramenta direta para o aumento do PIB.

Este estudo teve como limitação a falta de possibilidade de comparação com outras variáveis macroeconômicas que pudessem contribuir de forma ainda mais significativa para a pesquisa. Indica-se, como proposta de futuros trabalhos, realizar um estudo entre o PIB, o desemprego e o desenvolvimento tecnológico ou a capacidade de inovação. 


\section{REFERÊNCIAS}

BRASIL, Lei complementar de 14 de dezembro de 2006. Brasília 2006. Disponível em: $<$ http://www.planalto.gov.br/ccivil_03/leis/LCP/Lcp123.htm>acesso em 22 março 2016, 22:18

CASTELLS, Manuel. A Crise e seus efeitos. $2^{\mathrm{a}}$. ed. Rio de Janeiro: Editora Paz e Terra, 2000. p.32.

CRESPO, Antônio Arnot. Estatística Fácil.19. ed. São Paulo: Saraiva, 2009.

DAVID, Lillien. Sectorial Shifts and Cyclical Unemployment. Journal of Political Economy, August 1982.DORNBUSCH, FISCHER e STARTZ, São Paulo: Mcgraw Hill Brasil, 2009.

DRUCKER, Peter F. Inovação e espírito empreendedor - Entrepreneurship: práticas e princípios. São Paulo: Pioneira, 1992.IBGE. Disponível em:

$<$ http://brasilemsintese.ibge.gov.br/contas-nacionais/pib-valores-correntes.html > Acesso em 26 de março 2016, 11:29

Cenário econômico 2015 a 2024. Série estudos econômicos. Disponível em: <http://www.epe.gov.br/mercado/Documents/S\%C3\%A9rie\%20Estudos\%20de\%20Ene rgia/DEA\%2012-15\%20NT\%20Cenario\%20economico\%202015-2024vf.pdf $>$ Acesso em 27 março 2016, 00:18

Os números da rotatividade do Brasil: um olhar sobre os dados da RAIS 20022013. Disponível em:

http://www.dieese.org.br/notaaimprensa/2014/numerosRotatividadeBrasil.pdf > Acesso em 05 de abril de 2016. 22:06

Disponível em:

$<$ http://seriesestatisticas.ibge.gov.br/series.aspx?no=11\&op=2\&vcodigo=SCN53\&t=pro duto-interno-brutobrvariacao-volume> Acesso em 10 de abril 2016. 11:42

$\begin{array}{cccc}\text { Empresa } & \text { Brasil. } & \text { Disponível em } & \text { em } \\ \text { brasil/docs/empresa_brasil_n110 } & \text { Acesso em } 23 \text { maio } 2016 .\end{array}$

. $<$ http://seriesestatisticas.ibge.gov.br/series.aspx $?$ no=11\&op=2\&vcodigo=SCN5

$2 \& \mathrm{t}=$ produto-interno-bruto-br-valores-correntes $>$ acesso em 26 maio de 2016

. $<$ http://www.sebrae.com.br/sites/PortalSebrae/ufs/mt/noticias/micro-e-

pequenas-empresas-geram-27-do-pib-do-

brasil,ad0fc70646467410VgnVCM2000003c74010aRCRD> acesso em 03 março de 2016

ENIAC Projetos, Guarulhos (SP),V.5, n.2, jun.- dez. 2016 
IPEA, Mercado de trabalho e implicação para o desenvolvimento >http://www.ipea.gov.br/agencia/images/stories/PDFs/livros/livros/liv ro_micro_pequenasempresas.pdf $>$ Acesso em 31 de março. 21:38

LARSON e FARBER, Ron e Betsy. Estatística Aplicada. 4a. ed. São Paulo: Pearson, 2012.

LAGES, V. N. Sistema Financeiro e as Micro e Pequenas Empresas: diagnósticos e perspectivas. 2. ed. Brasília: SEBRAE, 2004.

NICHOLAS, N. GREGORY MANKIW. Introdução à Economia. 5a . ed. São Paulo: Cengage Learning, 2011.

PARKIN, Michael. Economia. 8a . ed. São Paulo: Pearson, 2009.

SACHS. Jeffrey. D. LARRAIN. B. FILIPE. Macroeconomia. São Paulo: Pearson Education, 2000

SAMUELSON P. A. e NORDHAUS W. Economia. $19^{\text {a }}$ ed. São Paulo: Mc Graw Hill, 2012.

SCHAUM, Murray R. Spiegel. Estatítica. $3^{\text {a }}$ ed. São Paulo: Pearson Makron Books, 1993.

SEBRAE<http://www.sebrae.com.br/sites/PortalSebrae/ufs/mt/noticias/Micro-epequenas-empresas-geram-27\%25-do-PIB-do-Brasil > acesso em 08 março 2016, 21:05

. $<$ https://www.google.com.br/url? $\mathrm{sa}=\mathrm{t} \& \mathrm{rct}=\mathrm{j} \& \mathrm{q}=\&$ esrc $=$ s \& source=web\&cd=1\& ved=0ahUKEwi9jMa2z9XLAhUGqx4KHXlLCmAQFggcMAA\&url=http\%3A\%2F\%2 Fobservatorio.sebraego.com.br\%2Fmidias\%2Fdownloads\%2F01042015153936.pdf\&us $\mathrm{g}=$ AFQjCNGdDFqXzYp7dwlvlt__HewCLmngTg\&sig2=pM3BiO-

fYZIVBb47GR1acQ\&bvm=bv.117218890,d.eWE\&cad=rja > acesso em 22 março 2016, 22:11

Serviço brasileiro de Apoio às Micro e Pequenas Empresas - Disponível em: $<$ http://www.ms.agenciasebrae.com.br/Estados/Novo\%20MPE\%20Indicadores\%20\%2023\%2007\%202014.pdf > Acesso em 06 de abril de 2016. 21:07

SKAF. Paulo. Onde estão as Micro e Pequenas Empresas no Brasil. $1^{\text {a }}$ ed. São Paulo: SEBRAE 2006. Disponível em <http://www.sebraesp.com.br/arquivos_site/biblioteca/EstudosPesquisas/mpes_numeros londe_mpes_brasil.pdf > Acesso em 04 de março de 2016. 11:04

KOTESKI. Marcos Antônio. As micro e pequenas empresas no contexto econômico brasileiro. Revista FAE Business. 2004. Disponível em

ENIAC Projetos, Guarulhos (SP),V.5, n.2, jun.- dez. 2016 
<http://www.fae.edu/publicacoes/pdf/revista_da_fae/fae_v8_n1/rev_fae_v8_n1_03_kot eski.pdf > acesso em 22 de março de 2016

STIGLITZ e WALSH. Joseph E. Carl E. Introdução à Macroeconomia. $3^{a}$ ed. São Paulo: Campus, 2003.

VIEIRA, M. L. A contribuição das micro e pequenas empresas para a redução da pobreza no Brasil. Dissertação (Mestrado Profissional) - Universidade Federal do Ceará, Curso de Pós-graduação em Economia, CAEN - Fortaleza, 2007.

VEJA. International Labour Office. Yearbook of Labour Statistics. Genebra, 1988.FEMICRO-ES < http://www.femicro-es.com.br/noticia/pesquisa-apontacrescimento-da-participacao-das-micro-e-pequenas-empresas-na-economia-brasileira $>$ acesso em 10 maio 2015. 